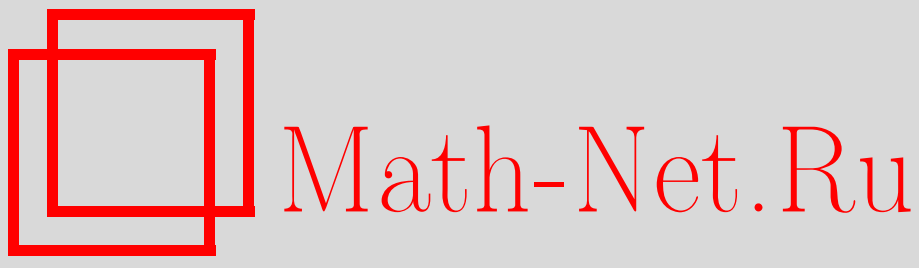

М. С. Сгибнев, Полумультипликативные оценки для решения многомерного уравнения восстановления, Изв. РАН. Сер. матем., 2002, том 66, выпуск 3, 159-174

DOI: https://doi.org/10.4213/im390

Использование Общероссийского математического портала Math-Net.Ru подразумевает, что вы прочитали и согласны с пользовательским соглашением

http://www . mathnet.ru/rus/agreement

Параметры загрузки:

IP : 34.229 .108 .108

26 апреля 2023 г., 08:32:33 
УДК 517.968.28+519.218.24

\author{
М. С. Сгибнев
}

\title{
Полумультипликативные оценки для решения многомерного уравнения восстановления
}

\begin{abstract}
Получены разложения для решения многомерного уравнения восстановления с полумультипликативными оценками для остатков. Учтено влияние корней характеристического уравнения на асимптотическое поведение решения. Полученные результаты применяются к исследованию асимптотики среднего числа частиц зависящих от возраста ветвящихся процессов с несколькими типами частиц.

Библиография: 13 наименований.
\end{abstract}

\section{$\S 1$. Введение}

Под уравнением многомерного восстановления понимается система уравнений (см. [1, гл. VIII, § 9])

$$
X_{i m}(x)=K_{i m}(x)+\sum_{j=1}^{n} \int_{0}^{x} X_{j m}(x-u) F_{i j}(d u),
$$

где $i=1, \ldots, n, m=1, \ldots, m_{1}, F_{i j}$ - неотрицательные конечные меры на $[0, \infty)$, $i, j=1, \ldots, n$, a $K_{i m}(x), i=1, \ldots, n, m=1, \ldots, m_{1},-$ измеримые, ограниченные в каждом конечном интервале функции. В матричной форме система уравнений (1) принимает вид

$$
\mathbf{X}(x)=\mathbf{K}(x)+\mathbf{F} * \mathbf{X}(x),
$$

где $\mathbf{X}(x), \mathbf{K}(x)$ - матрищы порядка $n \times m_{1}$, а $\mathbf{F}$ - матрица порядка $n \times n$ с элементами $X_{i m}(x), K_{i m}(x), F_{i j}$ соответственно; общим элементом матрищы $\mathbf{F} * \mathbf{X}(x)$ служит сумма, стоящая в правой части (1).

В настоящей работе предполагается, что $K_{i m}(x)$ - непрерывные справа функции ограниченной вариации на $[0, \infty)$. Каждая такая функция естественным образом порождает конечную вешественнозначную меру на $[0, \infty)$, которую будем обозначать той же буквой с теми же индексами. Будем искать решение системы (1) в классе непрерывных справа функций $X_{i m}(x)$, имеющих ограниченную вариацию на каждом конечном отрезке, или, что то же, в классе $\sigma$-конечных мер $X_{i m}$ на $[0, \infty)$, для которых $X_{i m}(x)$ суть их функции распределения: $X_{i m}(x)=$ $X_{i m}([0, x])$.

Уравнения многомерного восстановления (системы уравнений восстановления) изучались многими авторами (см. библиографию в [2], а также работы [1], [3]-[5]).

Работа выполнена при поддержке Российского фонда фундаментальных исследований (грант № 99-01-00504).

(C) М.С. Сгивнев, 2002 
Для произвольной комплекснозначной меры $\nu$ определим ее преобразование Лапласа

$$
\hat{\nu}(s)=\int_{\mathbb{R}} e^{s x} \nu(d x)
$$

для тех значений $s \in \mathbb{C}$, при которых данный интеграл имеет смысл, т.е. сходится абсолютно относительно полной вариации $|\nu|$ меры $\nu$. Условимся о том, что операции над матрицами осуществляются по-элементно; например,

$$
\int_{0}^{\infty} x \mathbf{F}(d x) \stackrel{\text { def }}{=}\left(\int_{0}^{\infty} x F_{i j}(d x)\right)
$$

а через $\widehat{\mathbf{F}}(s)$ будем обозначать матрицу, состояющую из преобразований Лапласа $\widehat{F}_{i j}(s)$ элементов матрищы $\mathbf{F}$. Аналогичное соглашение относится и к неравенствам, в которых участвуют матрицы. Пусть $\mathbf{I}-$ единичная матрица порядка $n \times n$.

Асимптотическое поведение решения $\mathbf{X}(x)$ уравнения (2) при $x \rightarrow \infty$ сушественно зависит от свойств матрищы $\mathbf{F}$. Настоящая работа посвящена изучению влияния корней характеристического уравнения

$$
\operatorname{det}(\mathbf{I}-\widehat{\mathbf{F}}(s))=0
$$

на асимптотику решения $\mathbf{X}(x)$ уравнения восстановления (2) при $x \rightarrow \infty$.

В качестве применения полученных результатов будет установлена асимптотика среднего числа частищ зависяших от возраста ветвяшихся процессов с несколькими типами частиц.

\section{§ 2. Предварительные сведения}

Пусть $\mathbf{B}$ - квадратная матрица порядка $n$ и $\mu_{1}, \ldots, \mu_{n}$ - ее собственные значения. Величина $\max _{1 \leqslant j \leqslant n}\left|\mu_{j}\right|$ называется спектральным радиусом В. По теореме Перрона-Фробениуса [6, теорема 9.2.1] любая неотрицательная неприводимая матрица $\mathbf{B}$ имеет положительное собственное значение $r$ кратности 1 , равное ее спектральному радиусу, и существует положительный правый (левый) собственный вектор, соответствуюший $r$. Если отказаться от условия неприводимости, то справедливо следуюшее утверждение [6, теорема 9.3.1, (i)]: любая неотрицательная матрица $\mathbf{B}$ имеет вешественное собственное значение $r$, равное спектральному радиусу В. В дальнейшем будем предполагать, что матрица $\widehat{\mathbf{F}}(0)=\left(\widehat{F}_{i j}(0)\right)=$ $\left(F_{i j}([0, \infty))\right)$ неприводима, а спектральный радиус матрицы $\mathbf{F}(\{0\})=\left(F_{i j}(\{0\})\right)$ меньше единицы.

В терминах преобразований Лапласа уравнение (2) можно записать в виде

$$
\widehat{\mathbf{X}}(s)=\widehat{\mathbf{K}}(s)+\widehat{\mathbf{F}}(s) \widehat{\mathbf{X}}(s) .
$$

Следовательно, $\widehat{\mathbf{X}}(s)=[\mathbf{I}-\widehat{\mathbf{F}}(s)]^{-1} \widehat{\mathbf{K}}(s) \stackrel{\text { def }}{=} \widehat{\mathbf{H}}(s) \widehat{\mathbf{K}}(s)$, где через $\widehat{\mathbf{H}}(s)$ обозначено преобразование Лапласа матричной меры восстановления

$$
\mathbf{H} \stackrel{\text { def }}{=} \sum_{k=0}^{\infty} \mathbf{F}^{k *}
$$


здесь $\mathbf{F}^{1 *} \stackrel{\text { def }}{=} \mathbf{F}, \mathbf{F}^{(k+1) *} \stackrel{\text { def }}{=} \mathbf{F}^{k *} * \mathbf{F}=\mathbf{F} * \mathbf{F}^{k *}, \mathbf{F}^{0 *} \stackrel{\text { def }}{=} \delta \mathbf{I}$, где $\delta-$ мера единичной массы, сосредоточенная в нуле. Таким образом, $\mathbf{X}=\mathbf{H} * \mathbf{K}$ или $\mathbf{X}(x)=$ $\int_{0}^{x} \mathbf{H}(d u) \mathbf{K}(x-u)$.

ОПРЕДЕЛЕНИЕ 1. Функция $\varphi(x), x \in \mathbb{R}$, называется полумультипликативной, если она конечна, положительна, измерима по Борелю и удовлетворяет следующим условиям: $\varphi(0)=1, \varphi(x+y) \leqslant \varphi(x) \varphi(y), x, y \in \mathbb{R}$.

Имеют место соотношения [7, теорема 7.6.2]

$$
\begin{aligned}
-\infty & <r_{-}(\varphi) \stackrel{\text { def }}{=} \lim _{x \rightarrow-\infty} \frac{\log \varphi(x)}{x}=\sup _{x<0} \frac{\log \varphi(x)}{x} \\
& \leqslant \inf _{x>0} \frac{\log \varphi(x)}{x}=\lim _{x \rightarrow \infty} \frac{\log \varphi(x)}{x} \stackrel{\text { def }}{=} r_{+}(\varphi)<\infty .
\end{aligned}
$$

Следующие примеры полумультипликативных функций на $\mathbb{R}_{+}$иллюстрируют широту понятия полумультипликативности: $\varphi(x)=(x+1)^{r}, r>0 ; \varphi(x)=$ $\exp \left(c x^{\beta}\right)$, где $c>0$ и $0<\beta<1 ; \varphi(x)=\exp \left(r_{+} x\right), r_{+}$вешественно. В первых двух случаях $r_{+}(\varphi)=0$, в то время как в последнем $r_{+}(\varphi)=r_{+}$. Произведение конечного числа полумультипликативных функций снова является полумультипликативной функцией. Кроме того, если $R(x), x \in \mathbb{R}_{+},-$положительная, начиная с некоторого места неубываюшая, правильно меняюшаяся на бесконечности функция с неотрицательным показателем $\beta$ (т.е. $R(t x) / R(x) \rightarrow t^{\beta}$ для $t>0$, когда $x \rightarrow \infty\left[8, \S\right.$ VIII.8]), то найдутся полумультипликативная функция $\varphi(x), x \in \mathbb{R}_{+}$, и точка $x_{0} \in(0, \infty)$ такие, что $c_{1} R(x) \leqslant \varphi(x) \leqslant c_{2} R(x)$ при всех $x \geqslant x_{0}$, где $c_{1}$ и $c_{2}$ - некоторые положительные постоянные [9, предложение].

Обозначим через $S(\varphi)$ совокупность всех комплекснозначных $\sigma$-конечных мер $\nu$, определенных на $\sigma$-алгебре $\mathscr{B}$ борелевских подмножеств прямой $\mathbb{R}$, таких, что

$$
\|\nu\|_{\varphi}=\int_{\mathbb{R}} \varphi(x)|\nu|(d x)<\infty .
$$

Совокупность $S(\varphi)$ - банахова алгебра с нормой $\|\cdot\|_{\varphi}$; операцией умножения элементов $\nu$ и $\kappa$ из $S(\varphi)$ служит их свертка $\nu * \kappa$, а единищей - мера $\delta$. (Теория банаховых алгебр $S(\varphi)$ для мер, сосредоточенных на $[0, \infty)$, изложена в [7, гл. 4].) Из (5) вытекает, что преобразование Лапласа $\hat{\nu}(s)$ произвольной меры $\nu \in S(\varphi)$ абсолютно сходится относительно полной вариации $|\nu|$ при $r_{-}(\varphi) \leqslant \operatorname{Re} s \leqslant r_{+}(\varphi)$. Следующие две теоремы о преобразованиях Лапласа доказаны в [10].

ТЕОРема 1. Пусть $\varphi(x), \quad x \in \mathbb{R},-$ полумультипликативная функиия такая, ито $r_{-}(\varphi)<r_{+}(\varphi)$, функиия $\varphi(x) / \exp \left[r_{+}(\varphi) x\right], \quad x \geqslant 0$, не убивает, а функиия $\varphi(x) / \exp \left[r_{-}(\varphi) x\right], \quad x \leqslant 0$, не возрастает. Предположим, что $\nu \in S(\varphi) u \hat{\nu}\left(s_{0}\right)=0$, , де $r_{-}(\varphi)<\operatorname{Re} s_{0}<r_{+}(\varphi)$. Тогда $\hat{\kappa}(s) \stackrel{\text { def }}{=} \hat{\nu}(s) /\left(s-s_{0}\right)$, $r_{-}(\varphi) \leqslant \operatorname{Re} s \leqslant r_{+}(\varphi),-$ преобразование Лапласа некоторой мерь $\kappa \in S(\varphi)$.

Если корень уравнения $\hat{\nu}(s)=0$ лежит на гранище полосы $\left\{r_{-}(\varphi) \leqslant \operatorname{Re} s \leqslant\right.$ $\left.r_{+}(\varphi)\right\}$, то справедлива следуюшая теорема (для определенности рассмотрим случай $\left.\operatorname{Re} s_{0}=r_{+}(\varphi)\right)$. 
ТЕОРема 2. Пусть $\varphi(x), \quad x \in \mathbb{R},-$ полумультипликативная функиия такая, что функция $\varphi(x) / \exp \left[r_{+}(\varphi) x\right], \quad x \geqslant 0$, не убввает, а $\varphi(x) / \exp \left[r_{-}(\varphi) x\right]$, $x \leqslant 0$, не возрастает. Пусть $\nu \in S(\varphi)$ и $\hat{\nu}\left(s_{0}\right)=0$, где $\operatorname{Re} s_{0}=r_{+}(\varphi)$. Предположим, что

$$
\int_{0}^{\infty} x \varphi(x)|\nu|(d x)<\infty \quad \text { uлu } \quad \int_{\mathbb{R}}|x| \varphi(x)|\nu|(d x)<\infty
$$

в зависимости от того, будет ли $r_{-}(\varphi)<r_{+}(\varphi)$ или $r_{-}(\varphi)=r_{+}(\varphi)$. Тогда $\hat{\kappa}(s) \stackrel{\text { def }}{=} \hat{\nu}(s) /\left(s-s_{0}\right), \quad r_{-}(\varphi) \leqslant \operatorname{Re} s \leqslant r_{+}(\varphi),-$ преобразование Лапласа некоторой меры $\kappa \in S(\varphi)$.

Абсолютно непрерывную компоненту произвольной неотрицательной меры $\nu$ обозначим через $\nu_{c}$, а сингулярную компоненту - через $\nu_{s}: \nu_{s}=\nu-\nu_{c}$. Если $\mathbf{F}-$ матрица, элементы которой суть неотрицательные меры, то через $\mathbf{F}_{s}$ будем обозначать матрицу, элементы которой суть сингулярные компоненты соответствующих элементов $\mathbf{F}$.

\section{§ 3. Теоремы многомерного восстановления}

Пусть $\varphi(x), \quad x \geqslant 0,-$ полумультипликативная функция такая, что $r_{+} \stackrel{\text { def }}{=}$ $r_{+}(\varphi) \geqslant 0$. Предположим, что $\widehat{\mathbf{F}}\left(r_{+}\right)<\infty$, и пусть множество $\mathscr{Z}$ корней характеристического уравнения (3), лежаших в полуплоскости $\left\{s \in \mathbb{C}: \operatorname{Re} s \leqslant r_{+}\right\}$, конечно: $\mathscr{Z}=\left\{s_{1}, \ldots, s_{l}\right\}$. Пусть $n_{j}-$ кратность корня $s_{j}$; это означает, что

$$
\operatorname{det}(\mathbf{I}-\widehat{\mathbf{F}}(s))=\left(s-s_{j}\right)^{n_{j}} f_{j}(s),
$$

где $f_{j}\left(s_{j}\right) \neq 0$. Если $\mathscr{Z} \neq \varnothing$, то среди элементов $\mathscr{Z}$ имеется один вешественный корень кратности единица, который мы будем обозначать через $q$; для определенности $q=s_{1}$ и $n_{1}=1$. Если $s \in \mathscr{Z}$, то $\bar{s} \in \mathscr{Z}$ и корень $\bar{s}$ имеет ту же кратность, что и $s$.

Пусть

$$
\int_{0}^{\infty} x^{2 n_{j}} e^{\operatorname{Re} s_{j} x} \mathbf{F}(d x)<\infty, \quad \int_{0}^{\infty} x^{n_{j}} e^{\operatorname{Re} s_{j} x}|\mathbf{K}|(d x)<\infty
$$

где $s_{j} \in \mathscr{Z}$. Определим коэффициенты $\mathbf{B}_{j k}, k=1, \ldots, n_{j}$, из асимптотического разложения

$$
\widehat{\mathbf{X}}(s)=[\mathbf{I}-\widehat{\mathbf{F}}(s)]^{-1} \widehat{\mathbf{K}}(s)=\sum_{k=1}^{n_{j}} \frac{(-1)^{k} \mathbf{B}_{j k}}{\left(s-s_{j}\right)^{k}}+o\left(\frac{1}{s-s_{j}}\right), \quad s \rightarrow s_{j} .
$$

Матричные коэффициенты $\mathbf{B}_{j k}$ зависят от матрицы $\mathbf{K}$, однако мы не будем фиксировать эту зависимость в обозначениях ради сокращения записи. Обозначим через $\mathscr{E}_{j}$ комплекснозначную меру с плотностью $\mathbf{1}_{(0, \infty)}(x) e^{-s_{j} x} ;$ здесь $\mathbf{1}_{A}(x)$ - индикаторная функция множества $A$. Преобразование Лапласа меры $\mathscr{E}_{j}$ равно $1 /\left(s_{j}-s\right)$, $\operatorname{Re}\left(s-s_{j}\right)<0$. 
Забегая вперед, сразу отметим, что искомое разложение для решения $\mathbf{X}$ будет иметь следующий вид:

$$
\mathbf{X}=\sum_{j=1}^{l} \sum_{k=1}^{n_{j}} \mathbf{B}_{j k}^{\mathscr{E}_{j}^{k *}}+\boldsymbol{\Delta}_{\mathbf{K}}
$$

с остатком $\boldsymbol{\Delta}_{\mathbf{K}}$, обладающим интегральной оценкой $\int_{0}^{\infty} \varphi(x)\left|\boldsymbol{\Delta}_{\mathbf{K}}\right|(d x)<\infty$ c полумультипликативной весовой функцией (здесь $\left|\boldsymbol{\Delta}_{\mathbf{K}}\right|$ - матрица полных вариаций вещественнозначных мер, являюшихся элементами матрицы $\left.\boldsymbol{\Delta}_{\mathbf{K}}\right)$. Это позволит получить оценку $o(1 / \varphi(x))$ для $\left|\boldsymbol{\Delta}_{\mathbf{K}}\right|((x, \infty))$ в соответствуюшем разложении для $\mathbf{X}(x)$ при $x \rightarrow \infty$.

Если $\mathscr{Z} \neq \varnothing$, то основной вклад в асимптотику $\mathbf{X}$ будет давать член $\mathbf{B}_{11} \mathscr{E} 1$, отвечающий корню $s_{1}=q$ уравнения (3). Поэтому целесообразно вычислить коэффициент $\mathbf{B}_{11}$ в явном виде.

Лемма 1. Пусть $\operatorname{det}(\mathbf{I}-\widehat{\mathbf{F}}(q))=0$. Предположим, ито $\widehat{\mathbf{F}}^{\prime}(q) \stackrel{\text { def }}{=}\left(\widehat{F}_{i j}^{\prime}(q)\right)-$ конечная матрица. Выберем левый и правый собственные векторы $\mathbf{l}=$ $\left(l_{1}, \ldots, l_{n}\right)$ u $\mathbf{r}=\left(r_{1}, \ldots, r_{n}\right)^{T}$ с положительными координатами, отвечающие собственному значению 1 матрицы $\widehat{\mathbf{F}}(q)$, так, чтобы $\mathbf{l} \mathbf{r}=1$. Тогда

$$
\mathbf{B}_{11}=\frac{\mathbf{r l} \widehat{\mathbf{K}}(q)}{\mathbf{l} \widehat{\mathbf{F}}^{\prime}(q) \mathbf{r}} .
$$

ДоКАЗАТЕЛЬСТво. Функция $\operatorname{det}(\mathbf{I}-\widehat{\mathbf{F}}(s))$ равна линейной комбинации произведений, состоящих из $n$ сомножителей, каждый из которых является преобразованием Лапласа некоторого элемента матрицы $\delta \mathbf{I}-\mathbf{F}$. Следовательно, $\operatorname{det}(\mathbf{I}-$ $\widehat{\mathbf{F}}(s))$ - преобразование Лапласа $\hat{\alpha}(s)$ некоторой вещественнозначной меры $\alpha$, причем функция $\hat{\alpha}(s)$ имеет производную в точке $s=q$. При $s \rightarrow q$ имеем $(\widehat{\mathbf{M}}(s)-$ присоединенная матрица к $\mathbf{I}-\widehat{\mathbf{F}}(s))$

$$
[\mathbf{I}-\widehat{\mathbf{F}}(s)]^{-1}=\frac{\widehat{\mathbf{M}}(s)}{\hat{\alpha}(s) /(s-q)} \frac{1}{s-q}=\frac{\widehat{\mathbf{M}}(q)}{\hat{\alpha}^{\prime}(q)} \frac{1}{s-q}+o\left(\frac{1}{s-q}\right)
$$

откуда

$$
[\mathbf{I}-\widehat{\mathbf{F}}(s)]^{-1} \widehat{\mathbf{K}}(s)=\frac{\widehat{\mathbf{M}}(q) \widehat{\mathbf{K}}(q)}{\hat{\alpha}^{\prime}(q)} \frac{1}{s-q}+o\left(\frac{1}{s-q}\right) .
$$

Таким образом, $\mathbf{B}_{11}=-\widehat{\mathbf{M}}(q) \widehat{\mathbf{K}}(q) / \hat{\alpha}^{\prime}(q)$. Равенство

$$
\frac{\widehat{\mathbf{M}}(q)}{\hat{\alpha}^{\prime}(q)}=-\frac{\mathbf{r l}}{\widehat{\mathbf{l}}^{\prime}(q) \mathbf{r}}
$$

нетрудно установить, модифицируя надлежащим образом рассуждения из доказательств лемм 8 и 6 в [11]. А именно, надо воспользоваться тем, что в нашем случае $\sum_{j=0}^{k} \widehat{\mathbf{F}}(q)^{j} / k \rightarrow \mathbf{r l}$ при $k \rightarrow \infty[12$, теорема 8.6.1], и заменить вектор-столбец $\mathbf{e}$ с единичными координатами на вектор $\mathbf{r}$. Для полноты изложения приведем соответствуюшие рассуждения. 
Имеем $\widehat{\mathbf{M}}(q)=c \mathbf{r l}$, где $c=\lim _{z \rightarrow 1-} \operatorname{det}(\mathbf{I}-z \widehat{\mathbf{F}}(q)) /(1-z)>0$. Действительно, при $0<z<1$ матрица $\widehat{\mathbf{M}}_{z}(q)$, присоединенная к $\mathbf{I}-z \widehat{\mathbf{F}}(q)$, равна

$$
\operatorname{det}(\mathbf{I}-z \widehat{\mathbf{F}}(q)) \sum_{k=0}^{\infty} z^{k} \widehat{\mathbf{F}}(q)^{k}=\frac{\operatorname{det}(\mathbf{I}-z \widehat{\mathbf{F}}(q))}{1-z} \cdot(1-z) \sum_{k=0}^{\infty} z^{k} \widehat{\mathbf{F}}(q)^{k}
$$

Характеристический многочлен $f(\lambda)$ матрицы $\widehat{\mathbf{F}}(q)$ равен

$$
f(\lambda) \stackrel{\text { def }}{=} \operatorname{det}(\lambda \mathbf{I}-\widehat{\mathbf{F}}(q))=\prod_{j=1}^{k}\left(\lambda-\lambda_{j}\right)^{m_{j}}
$$

где $\lambda_{j}$ - собственные числа $\widehat{\mathbf{F}}(q)$, а $m_{j}$ - их кратности, $j=1, \ldots, k, \sum_{j=1}^{k} m_{j}=n$, при этом собственное число $\lambda_{1}=1$ имеет кратность 1 . Поэтому

$$
\operatorname{det}(\mathbf{I}-z \widehat{\mathbf{F}}(q))=z^{n} f(1 / z)=z^{n} \prod_{j=1}^{k}\left(1 / z-\lambda_{j}\right)^{m_{j}}=(1-z) \prod_{j=2}^{k}\left(1-z \lambda_{j}\right)^{m_{j}}
$$

Таким образом, $c=\prod_{j=2}^{k}\left(1-\lambda_{j}\right)^{m_{j}}>0$, ибо $\lambda_{j} \neq 1 \forall j>1$, и если $\lambda_{j}-$ комплексное собственное число, то $\exists \lambda_{i}=\overline{\lambda_{j}}$ и $m_{i}=m_{j}$, откуда $\left(1-\lambda_{j}\right)^{m_{j}} \times$ $\left(1-\lambda_{i}\right)^{m_{i}}=\left|1-\lambda_{j}\right|^{2 m_{j}}$. В силу тауберовой теоремы для степенных рядов

$$
(1-z) \sum_{k=0}^{\infty} z^{k} \widehat{\mathbf{F}}(q)^{k} \rightarrow \mathbf{r l} \text { при } z \rightarrow 1-
$$

(см. $[8$, гл. XIII, $\S 5$, теорема 5]). Поэтому из (8) вытекает

$$
\widehat{\mathbf{M}}(q)=\lim _{z \rightarrow 1-} \widehat{\mathbf{M}}_{z}(q)=c \mathbf{r l}
$$

Продифференщируем равенство $[\mathbf{I}-\widehat{\mathbf{F}}(s)] \widehat{\mathbf{M}}(s)=\hat{\alpha}(s) \mathbf{I}$ в точке $s=q$. Получим

$$
-\widehat{\mathbf{F}}^{\prime}(q) \widehat{\mathbf{M}}(q)+[\mathbf{I}-\widehat{\mathbf{F}}(q)] \widehat{\mathbf{M}}^{\prime}(q)=\hat{\alpha}^{\prime}(q) \mathbf{I}
$$

Умножим обе части последнего равенства сначала слева на вектор $\mathbf{1}$, а затем справа на $\mathbf{r}$. В итоге, учитывая (9) и равенство $\mathbf{l r}=1$, получим

$$
-\mathbf{l} \widehat{\mathbf{F}}^{\prime}(q) \widehat{\mathbf{M}}(q) \mathbf{r}=-\mathbf{l} \widehat{\mathbf{F}}^{\prime}(q) c \mathbf{r}=\hat{\alpha}^{\prime}(q)
$$

Лемма доказана. 
ТЕОРема 3. Пусть $\varphi(x), \quad x \in \mathbb{R},-$ полумультипликативная функиия такая, что $\varphi(x) \equiv 1$ при $x<0, \quad r_{+} \geqslant 0$ и функиия $\varphi(x) / \exp \left(r_{+} x\right), \quad x \geqslant 0$, не убьвает. Предположим, что $\widehat{\mathbf{F}}\left(r_{+}\right)<\infty$ и спектральный радиус матриць $\left(\mathbf{F}^{m *}\right)_{s}^{\wedge}\left(r_{+}\right)$меньше единицы при некотором иелом $m \geqslant 1$. Допустим, что множество $\mathscr{Z}$ корней характеристического уравнения (3), лежащих в полуплоскости $\left\{s \in \mathbb{C}: \operatorname{Re} s \leqslant r_{+}\right\}$, конечно. Обозначим через $N$ максимальную кратность корней уравнения (3), лежащих на прямой $\left\{s \in \mathbb{C}: \operatorname{Re} s=r_{+}\right\}$ $(N=0$ означает, что таких корней на указанной прямой нет). Пусть такsce

$$
\int_{0}^{\infty} x^{2 N} \varphi(x) \mathbf{F}(d x)<\infty, \quad \int_{0}^{\infty} x^{N} \varphi(x)|\mathbf{K}|(d x)<\infty .
$$

Тогда для решения $\mathbf{X}$ уравнения (2) справедливо представление (7), в котором остаток $\boldsymbol{\Delta}_{\mathbf{K}}$ удовлетворяет неравенству $\int_{0}^{\infty} \varphi(x)\left|\boldsymbol{\Delta}_{\mathbf{K}}\right|(d x)<\infty$.

ДокАЗАТЕЛЬСтво. Выберем число $r_{-} \leqslant 0$ так, чтобы все корни характеристического уравнения (3) лежали в полосе $\left\{s \in \mathbb{C}: r_{-} \leqslant \operatorname{Re} s \leqslant r_{+}\right\}$. Сразу же оговоримся: параметр $r_{-}$играет вспомогательную роль и произвольность выбора $r_{-}$не повлияет на окончательные результаты настоящей работы. Образуем следующую систему полумультипликативньх функций: $\varphi_{k}(x) \stackrel{\text { def }}{=}(1+x)^{k} \varphi(x)$ при $x \geqslant 0$ и $\varphi_{k}(x) \stackrel{\text { def }}{=} \exp \left(r_{-} x\right)$ при $x<0$, где $k$ меняется от 0 до $2 N$. Очевидно, что $r_{+}\left(\varphi_{k}\right)=r_{+}$и $r_{-}\left(\varphi_{k}\right)=r_{-}$при всех $k=0, \ldots, 2 N$. Выберем $a>r_{+}$и положим $p=\sum_{j=1}^{l} n_{j}$. Рассмотрим функцию

$$
d(s) \stackrel{\text { def }}{=} \frac{(s-a)^{p} \operatorname{det}(\mathbf{I}-\widehat{\mathbf{F}}(s))}{\prod_{j=1}^{l}\left(s-s_{j}\right)^{n_{j}}}=\frac{(s-a)^{p} \hat{\alpha}(s)}{\prod_{j=1}^{l}\left(s-s_{j}\right)^{n_{j}}} .
$$

Доказательство теоремы 3 ввиду его большого объема удобно разбить на несколько лемм.

Лемма 2. Пусть выполнены условия теоремы 3. Тогда функиия $d(s)-$ преобразование Лапласа $\widehat{D}(s)$ некоторой меры $D$ из $S\left(\varphi_{N}\right)$.

ДокАЗАТЕЛЬСтво. Разлагая рациональную функцию на простые дроби, получим

$$
d(s)=\hat{\alpha}(s)+\sum_{j=1}^{l} \sum_{k=1}^{n_{j}} \frac{C_{j k}}{\left(s-s_{j}\right)^{k}} \hat{\alpha}(s),
$$

где $C_{j k}$ - константы. Из условий теоремы вытекает, что мера $\alpha$ с преобразованием Лапласа $\hat{\alpha}(s)=\operatorname{det}(\mathbf{I}-\widehat{\mathbf{F}}(s))$ принадлежит банаховой алгебре $S\left(\varphi_{2 N}\right)$. Применяя к каждому слагаемому $C_{j k} \hat{\alpha}(s) /\left(s-s_{j}\right)^{k}$ теорему 1 или теорему 2 , в зависимости от того, будет ли Re $s_{j}$ меньше или равно $r_{+}$, устанавливаем, что оно является преобразованием Лапласа некоторой меры из $S\left(\varphi_{2 N}\right)$ или $S\left(\varphi_{2 N-k}\right)$ соответственно. В итоге получим, что $d(s)=\widehat{D}(s)$, где $D \in S\left(\varphi_{N}\right)$. Лемма доказана.

ЛЕмма 3. Пусть выполнены условия теоремы 3 . Элемент $D \in S\left(\varphi_{N}\right)$ обратим в $S\left(\varphi_{N}\right)$. 
ДоКАЗАТЕЛЬСТВО в значительной степени повторяет рассуждения из $[10$, теорема 4], однако в связи с матричной спецификой рассматриваемого случая приведем его в полном объеме. Пусть $\mathscr{M}$ - пространство максимальных идеалов алгебры $S\left(\varphi_{N}\right)$. Каждый максимальный идеал $M \in \mathscr{M}$ порождает некоторый гомоморфизм $h: S\left(\varphi_{N}\right) \rightarrow \mathbb{C}$, и $M-$ ядро этого гомоморфизма. Обозначим через $\nu(M)$ значение $h$ на $\nu \in S\left(\varphi_{N}\right)$. Элемент $\nu \in S\left(\varphi_{N}\right)$ имеет обратный тогда и только тогда, когда $\nu$ не принадлежит никакому максимальному идеалу $M \in \mathscr{M}$. Иными словами, $\nu$ обратим тогда и только тогда, когда $\nu(M) \neq 0$ при любом $M \in \mathscr{M}$.

Пространство $\mathscr{M}$ разбивается на два множества: множество максимальных идеалов, не содержащих совокупности $L\left(\varphi_{N}\right)$ всех абсолютно непрерывных мер из $S\left(\varphi_{N}\right)$, и $\mathscr{M}_{2}=\mathscr{M} \backslash \mathscr{M}_{1}$. Если $M \in \mathscr{M}_{1}$, то порожденный им гомоморфизм имеет вид $h(\nu)=\hat{\nu}\left(s_{0}\right), r_{-} \leqslant \operatorname{Re} s_{0} \leqslant r_{+}$. В этом случае $M=\left\{\nu \in S\left(\varphi_{N}\right): \hat{\nu}\left(s_{0}\right)=0\right\}[7$, гл. IV,$\S 4]$. Если $M \in \mathscr{M}_{2}$, то $\nu(M)=0 \forall \nu \in L\left(\varphi_{N}\right)$.

Покажем, что $D(M) \neq 0$ для любого $M \in \mathscr{M}$, тем самым мы установим существование обратного элемента $D^{-1} \in S\left(\varphi_{N}\right)$. Действительно, если $M \in \mathscr{M}_{1}$, то при некотором $s_{0} \in\left\{r_{-} \leqslant \operatorname{Re} s \leqslant r_{+}\right\}$имеем $D(M)=\widehat{D}\left(s_{0}\right) \neq 0$.

Пусть теперь $M \in \mathscr{M}_{2}$. По теореме о строении произвольного гомоморфизма $h: S\left(\varphi_{N}\right) \rightarrow \mathbb{C}[13$, теорема 1$]$

$$
h(\nu)=\int_{\mathbb{R}} \chi(x, \nu) \exp (\beta x) \nu(d x), \quad \nu \in S\left(\varphi_{N}\right),
$$

где $\beta$ - вещественное число такое, что $r_{-} \leqslant \beta \leqslant r_{+}$, а функция $\chi(x, \nu)$ двух переменных $x \in \mathbb{R}$ и $\nu \in S\left(\varphi_{N}\right)$ - обобшенный характер, из свойств которого нам потребуется лишь одно: $|\nu|-\operatorname{ess}_{\sup } \in \mathbb{R}|\chi(x, \nu)| \leqslant 1$. В силу мультипликативности функционала $\nu \rightarrow \nu(M), \nu \in S\left(\varphi_{N}\right)$, имеем $\mathbf{F}(M)^{m}=\mathbf{F}^{m *}(M)=\left(\mathbf{F}^{m *}\right)_{s}(M)$. Обозначим, для краткости, $\boldsymbol{\Theta}=\left(\Theta_{i j}\right) \stackrel{\text { def }}{=}\left(\mathbf{F}^{m *}\right)_{s}$.

Спектральньй радиус произвольной матрицы $\mathbf{B}$ равен $\lim _{k \rightarrow \infty}\left\|\mathbf{B}^{k}\right\|^{1 / k}$, где $\left\|\mathbf{B}^{k}\right\|$ - евклидова норма матрицы $\mathbf{B}^{k}$. Поэтому если элементы одной матрицы не превосходят по абсолютной величине соответствуюших элементов некоторой неотрицательной матрищы, то спектральный радиус первой матрищы не превосходит спектрального радиуса второй. В силу (11) при некотором $\beta \in\left[r_{-}, r_{+}\right]$имеем

$$
\begin{aligned}
\left|\Theta_{i j}(M)\right| & =\left|\int_{\mathbb{R}} \chi\left(x, \Theta_{i j}\right) \exp (\beta x) \Theta_{i j}(d x)\right| \\
& \leqslant \int_{\mathbb{R}} \exp (\beta x) \Theta_{i j}(d x) \leqslant \int_{\mathbb{R}} \exp \left(r_{+} x\right) \Theta_{i j}(d x),
\end{aligned}
$$

откуда вытекает, что спектральный радиус матрицы $\Theta(M)$ не превосходит спектрального радиуса матрицы $\widehat{\boldsymbol{\Theta}}\left(r_{+}\right)=\left(\mathbf{F}^{m *}\right)_{s}^{\wedge}\left(r_{+}\right)$, которьй по предположению меньше единицы. Следовательно, спектральный радиус матрицы $\mathbf{F}(M)^{m}$ меньше единицы. Поэтому спектральный радиус матрицы $\mathbf{F}(M)$, равный корню степени $m$ из спектрального радиуса матрицы $\mathbf{F}(M)^{m}$, также будет меньше единицы. Поскольку $T\left(s_{j}\right)^{k} F \in L\left(\varphi_{N}\right) \forall j, k$, из (10) вытекает, что $D(M)=\operatorname{det}(\mathbf{I}-\mathbf{F}(M)) \neq 0$.

Итак, $D(M) \neq 0$ для любого $M \in \mathscr{M}$. Это означает, что $\exists D^{-1} \in S\left(\varphi_{N}\right)$ и функция $1 / d(s), r_{-} \leqslant \operatorname{Re} s \leqslant r_{+},-$преобразование Лапласа элемента $D^{-1}$. Лемма доказана. 
Рассмотрим вспомогательную матрицу

$$
\mathbf{q}(s) \stackrel{\text { def }}{=} \frac{\prod_{j=1}^{l}\left(s-s_{j}\right)^{n_{j}}}{(s-a)^{p}}[\mathbf{I}-\widehat{\mathbf{F}}(s)]^{-1}, \quad s \in\left\{r_{-} \leqslant \operatorname{Re} s \leqslant r_{+}\right\} \backslash \mathscr{Z} .
$$

Лемма 4. Пусть выполнены условия теоремы 3. Тогда $\mathbf{q}(s)$ является

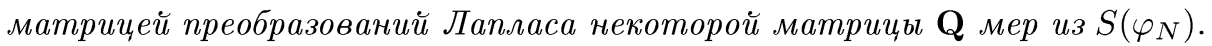

ДоказАТЕЛЬСтво. Напомним, что $\widehat{\mathbf{M}}(s)$ - присоединенная матрища к $\mathbf{I}-\widehat{\mathbf{F}}(s)$. Тогда $\mathbf{q}(s)=[1 / d(s)] \widehat{\mathbf{M}}(s)$. Следовательно, по лемме $3 \mathbf{q}(s)=\widehat{\mathbf{Q}}(s)$, где $\mathbf{Q} \stackrel{\text { def }}{=}$ $D^{-1} * \mathbf{M} \in S\left(\varphi_{N}\right)$ (элементы матрицы $\mathbf{Q}$ суть свертки меры $D^{-1}$ с соответствуюшими элементами матрищы $\mathbf{M}$, принадлежашими $\left.S\left(\varphi_{2 N}\right) \subset S\left(\varphi_{N}\right)\right)$. Лемма доказана.

Вернемся к доказательству теоремы 3. Имеем

$$
\begin{aligned}
\widehat{\mathbf{X}}(s) & =[\mathbf{I}-\widehat{\mathbf{F}}(s)]^{-1} \widehat{\mathbf{K}}(s)=\frac{(s-a)^{p}}{\prod_{j=1}^{l}\left(s-s_{j}\right)^{n_{j}}} \widehat{\mathbf{Q}}(s) \widehat{\mathbf{K}}(s) \\
& =\left[1+\sum_{j=1}^{l} \sum_{k=1}^{n_{j}} \frac{C_{j k}}{\left(s-s_{j}\right)^{k}}\right] \widehat{\mathbf{Q}}(s) \widehat{\mathbf{K}}(s)
\end{aligned}
$$

(здесь мы снова воспользовались разложением рациональной функции на простые дроби). Положим для краткости $\mathbf{W} \stackrel{\text { def }}{=} \mathbf{Q} * \mathbf{K}$. Очевидно, что $\mathbf{W} \in S\left(\varphi_{N}\right)$. Осуществим следующие преобразования:

$$
\frac{\widehat{\mathbf{W}}(s)}{\left(s-s_{j}\right)^{k}}=\frac{\widehat{\mathbf{W}}\left(s_{j}\right)}{\left(s-s_{j}\right)^{k}}+\frac{\widehat{\mathbf{W}}(s)-\widehat{\mathbf{W}}\left(s_{j}\right)}{\left(s-s_{j}\right)^{k}}=\sum_{i=0}^{k-1} \frac{\mathbf{w}_{i, j}\left(s_{j}\right)}{\left(s-s_{j}\right)^{k-i}}+\mathbf{w}_{k, j}(s)
$$

где

$$
\mathbf{w}_{0, j}(s) \stackrel{\text { def }}{=} \widehat{\mathbf{W}}(s), \quad \mathbf{w}_{i, j}(s) \stackrel{\text { def }}{=} \frac{\mathbf{w}_{i-1, j}(s)-\mathbf{w}_{i-1, j}\left(s_{j}\right)}{s-s_{j}}, \quad i=1, \ldots, k .
$$

Применяя теорему 1 или теорему 2, в зависимости от того, будет ли $\operatorname{Re} s_{j}$ меньше или равно $r_{+}$, видим, что матричная мера $\mathbf{W}_{i, j} \stackrel{\text { def }}{=} T\left(s_{j}\right)^{i} \mathbf{W}$ с преобразованием Лапласа $\mathbf{w}_{i, j}(s)$ принадлежит банаховой алгебре $S\left(\varphi_{N}\right)$ или $S\left(\varphi_{N-k}\right)$ соответственно. Подставляя (13) в (12) и производя суммирование, получим в силу единственности разложения (6)

$$
\left[1+\sum_{j=1}^{l} \sum_{k=1}^{n_{j}} \frac{C_{j k}}{\left(s-s_{j}\right)^{k}}\right] \widehat{\mathbf{W}}(s)=\widehat{\mathbf{W}}(s)+\sum_{j=1}^{l} \sum_{k=1}^{n_{j}} \frac{(-1)^{k} \mathbf{B}_{j k}}{\left(s-s_{j}\right)^{k}}+\sum_{j=1}^{l} \sum_{k=1}^{n_{j}} C_{j k} \mathbf{w}_{k, j}(s) .
$$

Положим $\boldsymbol{\Delta}_{\mathbf{K}} \stackrel{\text { def }}{=} \mathbf{W}+\sum_{j=1}^{l} \sum_{k=1}^{n_{j}} C_{j k} T\left(s_{j}\right)^{k} \mathbf{W}$. Тогда $\boldsymbol{\Delta}_{\mathbf{K}} \in S\left(\varphi_{0}\right)=S(\varphi)$ и

$$
\widehat{\mathbf{X}}(s)=\sum_{j=1}^{l} \sum_{k=1}^{n_{j}} \frac{(-1)^{k} \mathbf{B}_{j k}}{\left(s-s_{j}\right)^{k}}+\hat{\boldsymbol{\Delta}}_{\mathbf{K}}(s)
$$

Переходя в этом равенстве от преобразований Лапласа к мерам, получим представление (7). Теорема 3 доказана. 
ЗАмЕчАНИЕ 1. Условие, состоящее в том, что при некотором целом $m \geqslant 1$ спектральный радиус $\rho_{m}$ матрицы $\mathbf{V}(m)=\left(V_{k j}(m)\right) \stackrel{\text { def }}{=}\left(\mathbf{F}^{m *}\right)_{s}^{\wedge}\left(r_{+}\right)$меньше единицы, является, вообше говоря, необходимым для того, чтобы остаток $\boldsymbol{\Delta}_{\mathbf{K}}$ в разложении (7) удовлетворял интегральной оценке

$$
\int_{0}^{\infty} e^{r_{+} x}\left|\boldsymbol{\Delta}_{\mathbf{K}}\right|(d x)<\infty .
$$

Действительно, допустим противное, т.е. что $\rho_{m} \geqslant 1 \forall m \geqslant 1$. Пусть $\mathbf{H}$ - матричная мера восстановления (4) $(\mathbf{H}-$ решение уравнения $(2)$ при $\mathbf{K}=\delta \mathbf{I})$. Предположим, что для $\mathbf{H}$ справедливо разложение (7) с оценкой $\int_{0}^{\infty} e^{r_{+} x}|\boldsymbol{\Delta}|(d x)<\infty$, где $\boldsymbol{\Delta}=\boldsymbol{\Delta}_{\mathbf{K}}$ при $\mathbf{K}=\delta \mathbf{I}$. Пусть $\mathbf{x}(m)=\left(x_{1}(m), \ldots, x_{n}(m)\right)$ - неотрицательный правый собственный вектор матрицы $\mathbf{V}(m)$, соответствующий собственному значению $\rho_{m}$, такой, что сумма его координат равна единице. Матрица $\mathbf{V}(m)$ содержит, по крайней мере, один элемент, не меньший $1 / n$. В самом деле, допустив противное, нетрудно прийти к противоречию: с одной стороны имеем

$$
\sum_{j=1}^{n}[\mathbf{V}(m) \mathbf{x}(m)]_{j}=\sum_{j=1}^{n} \sum_{k=1}^{n} V_{j k}(m) x_{k}(m)<\sum_{j=1}^{n} \sum_{k=1}^{n} \frac{1}{n} x_{k}(m)=1,
$$

а с другой -

$$
\sum_{j=1}^{n}[\mathbf{V}(m) \mathbf{x}(m)]_{j}=\rho_{m} \sum_{j=1}^{n} x_{j}(m)=\rho_{m} \geqslant 1 .
$$

Таким образом, найдутся пара индексов $(j, k)$ и целочисленная последовательность $\left\{m_{i}\right\}\left(m_{i} \rightarrow \infty, i \rightarrow \infty\right)$ такие, что у матриц $\mathbf{V}\left(m_{i}\right)$ на пересечении $j$-й строки и $k$-го столбца стоят элементы, не меньшие $1 / n$. Выберем множества $A_{m_{i}} \in \mathscr{B}$ лебеговой меры нуль таким образом, чтобы мера $V_{j k}\left(m_{i}\right)$ была сосредоточена на $A_{m_{i}}$. Тогда лебегова мера множества $A \stackrel{\text { def }}{=} \bigcup_{i} A_{m_{i}}$ равна нулю и из разложения (7) для $\mathbf{X}=\mathbf{H}$ вытекает, что справа в матричном неравенстве

$$
\int_{A} e^{r_{+} x} \mathbf{H}(d x) \geqslant \sum_{i=1}^{n} \int_{A} e^{r_{+} x}\left(\mathbf{F}^{m_{i} *}\right)_{s}(d x)
$$

на пересечении $j$-й строки и $k$-го столбца стоит $+\infty$, а в силу $(7)$

$$
\int_{A} e^{r_{+} x} \mathbf{H}(d x) \leqslant \int_{A} e^{r_{+} x}|\boldsymbol{\Delta}|(d x)<\infty .
$$

Полученное противоречие показывает, что если для $\mathbf{H}$ справедливо разложение (7) с оценкой $\int_{0}^{\infty} e^{r+x}|\boldsymbol{\Delta}|(d x)<\infty$, то тогда обязательно найдется целое $m \geqslant 1$ такое, что спектральный радиус матрицы $\left(\mathbf{F}^{m *}\right)_{s}^{\wedge}\left(r_{+}\right)$строго меньше единицы.

СлеДСТВИЕ 1. Пусть выполнень условия теоремы $3 u \operatorname{det}(\mathbf{I}-\widehat{\mathbf{F}}(0)) \neq 0$. Тогда для решения $\mathbf{X}(x)$ уравнения (2) справедливо разложение

$$
\mathbf{X}(x)=[\mathbf{I}-\widehat{\mathbf{F}}(0)]^{-1} \widehat{\mathbf{K}}(0)-\sum_{j=1}^{l} \sum_{k=1}^{n_{j}} \mathbf{B}_{j k} e^{-s_{j} x} \sum_{p=0}^{k-1} \frac{x^{p}}{p ! s_{j}^{k-p}}-\boldsymbol{\Delta}_{\mathbf{K}}((x, \infty)),
$$

२дe

$$
\left|\boldsymbol{\Delta}_{\mathbf{K}}\right|((x, \infty))=o(1 / \varphi(x)), \quad x \rightarrow \infty .
$$


ДокАЗАТЕЛЬСтво. Из (7) вытекает

$$
\mathbf{X}(x)=\mathbf{X}([0, x])=\sum_{j=1}^{l} \sum_{k=1}^{n_{j}} \mathbf{B}_{j k} \mathscr{E}_{j}^{k *}([0, x])+\boldsymbol{\Delta}_{\mathbf{K}}([0, \infty))-\boldsymbol{\Delta}_{\mathbf{K}}((x, \infty)) .
$$

Имеем

$$
\begin{aligned}
\boldsymbol{\Delta}_{\mathbf{K}}([0, \infty)) & =\lim _{s \rightarrow 0}\left\{[\mathbf{I}-\widehat{\mathbf{F}}(s)]^{-1} \widehat{\mathbf{K}}(s)-\sum_{j=1}^{l} \sum_{k=1}^{n_{j}} \frac{(-1)^{k} \mathbf{B}_{j k}}{\left(s-s_{j}\right)^{k}}\right\} \\
& =[\mathbf{I}-\widehat{\mathbf{F}}(0)]^{-1} \widehat{\mathbf{K}}(0)-\sum_{j=1}^{l} \sum_{k=1}^{n_{j}} \frac{\mathbf{B}_{j k}}{s_{j}^{k}} .
\end{aligned}
$$

Далее,

$$
\mathscr{E}_{j} k *([0, x])=-e^{-s_{j} x} \sum_{p=0}^{k-1} \frac{x^{p}}{p ! s_{j}^{k-p}}+\frac{1}{s_{j}^{k}} .
$$

Равенство (14) установлено. Оценка (15) вытекает из очевидного соотношения

$$
\varphi(x)\left|\boldsymbol{\Delta}_{\mathbf{K}}\right|((x, \infty)) \leqslant \int_{x}^{\infty} \varphi(y)\left|\boldsymbol{\Delta}_{\mathbf{K}}\right|(d y) \rightarrow 0, \quad x \rightarrow \infty .
$$

Следствие доказано.

СледСТвиЕ 2. Пусть выполнены условия теоремы $3, \operatorname{det}(\mathbf{I}-\widehat{\mathbf{F}}(0))=0 u$ $\widehat{\mathbf{M}}(s)$ - присоединенная матрица $\kappa \mathbf{I}-\widehat{\mathbf{F}}(s)$. Выберем левый и правый собственные векторы $\mathbf{l}$ и $\mathbf{r}$ с положительными координатами, отвечающие собственному значению 1 матрицы $\widehat{\mathbf{F}}(0)$, так, чтобы $\mathbf{l} \mathbf{r}=1$. Пусть $s_{1}=0$. Тогда для решения $\mathbf{X}(x)$ уравнения (2) справедливо разложение

$$
\begin{aligned}
\mathbf{X}(x)= & \frac{\mathbf{r l} \widehat{\mathbf{K}}(0)}{\mathbf{l} \widehat{\mathbf{F}}^{\prime}(0) \mathbf{r}} x+\left[\frac{\widehat{\mathbf{M}}^{\prime}(0)}{\hat{\alpha}^{\prime}(0)}-\frac{\widehat{\mathbf{M}}(0) \hat{\alpha}^{\prime \prime}(0)}{2 \hat{\alpha}^{\prime}(0)^{2}}\right] \widehat{\mathbf{K}}(0)-\frac{\mathbf{r l} \widehat{\mathbf{K}}^{\prime}(0)}{\mathbf{l}^{\prime}(0) \mathbf{r}} \\
& -\sum_{j=2}^{l} \sum_{k=1}^{n_{j}} \mathbf{B}_{j k} e^{-s_{j} x} \sum_{p=0}^{k-1} \frac{x^{p}}{p ! s_{j}^{k-p}}-\boldsymbol{\Delta}_{\mathbf{K}}((x, \infty)),
\end{aligned}
$$

где $\boldsymbol{\Delta}_{\mathbf{K}}((x, \infty))$ удовлетворяет (15).

ДокАЗАТЕльСТво. Чтобы избежать путаницы в доказательстве, будем фиксировать графически зависимость коэффициентов $\mathbf{B}_{j k}$ от $\mathbf{K}$, поскольку здесь нам предстоит рассматривать отдельно как общий случай с произвольной матрицей $\mathbf{K}$, так и частный случай $\mathbf{K}=\delta \mathbf{I}$, соответствуюший матричной мере восстановления. Имеем

$$
\begin{gathered}
\mathbf{X}(x)=\mathbf{B}_{11}(\mathbf{K}) x+\sum_{j=2}^{l} \sum_{k=1}^{n_{j}} \mathbf{B}_{j k}(\mathbf{K}) \mathscr{E}_{j}^{k *}([0, x])+\boldsymbol{\Delta}_{\mathbf{K}}([0, \infty))-\boldsymbol{\Delta}_{\mathbf{K}}((x, \infty)) \\
\boldsymbol{\Delta}_{\mathbf{K}}([0, \infty))=\lim _{s \rightarrow 0}\left\{[\mathbf{I}-\widehat{\mathbf{F}}(s)]^{-1} \widehat{\mathbf{K}}(s)+\frac{\mathbf{B}_{11}(\mathbf{K})}{s}-\sum_{j=2}^{l} \sum_{k=1}^{n_{j}} \frac{(-1)^{k} \mathbf{B}_{j k}(\mathbf{K})}{\left(s-s_{j}\right)^{k}}\right\} \\
=\lim _{s \rightarrow 0}\left\{[\mathbf{I}-\widehat{\mathbf{F}}(s)]^{-1} \widehat{\mathbf{K}}(s)+\frac{\mathbf{B}_{11}(\mathbf{K})}{s}\right\}-\sum_{j=2}^{l} \sum_{k=1}^{n_{j}} \frac{\mathbf{B}_{j k}(\mathbf{K})}{s_{j}^{k}}
\end{gathered}
$$


В обшем случае предел

$$
\mathbf{D}_{\mathbf{K}} \stackrel{\text { def }}{=} \lim _{s \rightarrow 0}\left\{[\mathbf{I}-\widehat{\mathbf{F}}(s)]^{-1} \widehat{\mathbf{K}}(s)+\frac{\mathbf{B}_{11}(\mathbf{K})}{s}\right\}
$$

нетрудно выразить через $\mathbf{D}_{\mathbf{K}}$ при $\mathbf{K}=\delta \mathbf{I}$. Действительно, так как $\mathbf{B}_{11}(\mathbf{K})=$ $\mathbf{B}_{11}(\delta \mathbf{I}) \widehat{\mathbf{K}}(0)$, имеем

$$
\begin{aligned}
{[\mathbf{I}-\widehat{\mathbf{F}}(s)]^{-1} \widehat{\mathbf{K}}(s)+\frac{\mathbf{B}_{11}(\mathbf{K})}{s}=} & \left\{[\mathbf{I}-\widehat{\mathbf{F}}(s)]^{-1}+\frac{\mathbf{B}_{11}(\delta \mathbf{I})}{s}\right\} \widehat{\mathbf{K}}(0) \\
& +[\mathbf{I}-\widehat{\mathbf{F}}(s)]^{-1}[\widehat{\mathbf{K}}(s)-\widehat{\mathbf{K}}(0)],
\end{aligned}
$$

откуда

$$
\mathbf{D}_{\mathbf{K}}=\mathbf{D}_{\delta \mathbf{I}} \widehat{\mathbf{K}}(0)+\lim _{s \rightarrow 0}[\mathbf{I}-\widehat{\mathbf{F}}(s)]^{-1}[\widehat{\mathbf{K}}(s)-\widehat{\mathbf{K}}(0)] .
$$

Предел в правой части равен

$$
\lim _{s \rightarrow 0} \frac{\widehat{\mathbf{M}}(s)}{\operatorname{det}(\mathbf{I}-\widehat{\mathbf{F}}(s)) / s} \frac{\widehat{\mathbf{K}}(s)-\widehat{\mathbf{K}}(0)}{s}=\frac{\widehat{\mathbf{M}}(0) \widehat{\mathbf{K}}^{\prime}(0)}{\hat{\alpha}^{\prime}(0)}=-\frac{\mathbf{r} \mathbf{l} \widehat{\mathbf{K}}^{\prime}(0)}{\mathbf{l} \widehat{\mathbf{F}}^{\prime}(0) \mathbf{r}} .
$$

Вычислим $\mathbf{D}_{\delta \mathbf{I}}$. Заметим, что $\mathbf{B}_{11}(\delta \mathbf{I})=-\widehat{\mathbf{M}}(0) / \hat{\alpha}^{\prime}(0)$. Далее, полагая $g(s) \stackrel{\text { def }}{=}$ $s / \hat{\alpha}(s), g(0) \stackrel{\text { def }}{=} 1 / \hat{\alpha}^{\prime}(0)$, имеем при $s \rightarrow 0$

$$
\begin{aligned}
{[\mathbf{I}-\widehat{\mathbf{F}}(s)]^{-1} } & =\frac{\widehat{\mathbf{M}}(s)}{\hat{\alpha}(s)}=\frac{\widehat{\mathbf{M}}(s) g(s)}{s} \\
& =\frac{1}{s}\left[\widehat{\mathbf{M}}(0)+\widehat{\mathbf{M}}^{\prime}(0) s+o(s)\right]\left[g(0)+g^{\prime}(0) s+o(s)\right] \\
& =\frac{1}{s} \frac{\widehat{\mathbf{M}}(0)}{\hat{\alpha}^{\prime}(0)}+\widehat{\mathbf{M}}(0) g^{\prime}(0)+\widehat{\mathbf{M}}^{\prime}(0) g(0)+o(1) .
\end{aligned}
$$

Таким образом,

$$
\mathbf{D}_{\delta \mathbf{I}}=\widehat{\mathbf{M}}(0) g^{\prime}(0)+\widehat{\mathbf{M}}^{\prime}(0) g(0)=-\frac{\widehat{\mathbf{M}}(0) \hat{\alpha}^{\prime \prime}(0)}{2 \hat{\alpha}^{\prime}(0)^{2}}+\frac{\widehat{\mathbf{M}}^{\prime}(0)}{\hat{\alpha}^{\prime}(0)} .
$$

Из (20)-(22) вытекает

$$
\mathbf{D}_{\mathbf{K}}=\left[\frac{\widehat{\mathbf{M}}^{\prime}(0)}{\hat{\alpha}^{\prime}(0)}-\frac{\widehat{\mathbf{M}}(0) \hat{\alpha}^{\prime \prime}(0)}{2 \hat{\alpha}^{\prime}(0)^{2}}\right] \widehat{\mathbf{K}}(0)+\frac{\widehat{\mathbf{M}}(0) \widehat{\mathbf{K}}^{\prime}(0)}{\hat{\alpha}^{\prime}(0)} .
$$

Подставляя найденное значение $\mathbf{D}_{\mathbf{K}}$ в (19), получим

$$
\boldsymbol{\Delta}_{\mathbf{K}}([0, \infty))=\left[\frac{\widehat{\mathbf{M}}^{\prime}(0)}{\hat{\alpha}^{\prime}(0)}-\frac{\widehat{\mathbf{M}}(0) \hat{\alpha}^{\prime \prime}(0)}{2 \hat{\alpha}^{\prime}(0)^{2}}\right] \widehat{\mathbf{K}}(0)+\frac{\widehat{\mathbf{M}}(0) \widehat{\mathbf{K}}^{\prime}(0)}{\hat{\alpha}^{\prime}(0)}-\sum_{j=2}^{l} \sum_{k=1}^{n_{j}} \frac{\mathbf{B}_{j k}(\mathbf{K})}{s_{j}^{k}} .
$$

Наконец, из (18) и (16) вытекает искомое разложение (17). Следствие доказано. 


\section{§ 4. Асимптотика среднего числа частиц}

В настояшем параграфе мы применим доказанные теоремы многомерного восстановления к исследованию асимптотики среднего числа частищ зависящих от возраста ветвящихся процессов. В самой общей модели таких процессов - модель I в терминологии [1] - предполагается, что популяция состоит из $n$ типов частиц. Напомним вкратце устройство этих процессов $[1$, гл. VIII]. Каждая частица $i$-го типа имеет случайную продолжительность жизни $\tau_{i}$ с невырожденной функцией распределения $G_{i}(t)$. В конще жизни частица $i$-го типа преврашается в совокупность частищ $\boldsymbol{\nu}_{i}=\left(\nu_{i 1}, \ldots, \nu_{i n}\right)$, где $\nu_{i k}$ - число частиц $k$-го типа нулевого возраста, порожденных частицей $i$-го типа в момент ее гибели. Обозначим $p_{i \boldsymbol{\alpha}}(u)=\mathbf{P}\left(\boldsymbol{\nu}_{i}=\boldsymbol{\alpha} \mid \tau_{i}=u\right)$, где $\boldsymbol{\alpha}=\left(\alpha_{1}, \ldots, \alpha_{n}\right)$ - вектор с неотрицательными целочисленньми координатами. Функции $p_{i \boldsymbol{\alpha}}(u)$ переменной $u$ предполагаются измеримыми по Борелю. Эволюция частицы $i$-го типа определяется совместным распределением

$$
\mathbf{P}\left(\tau_{i} \in B, \boldsymbol{\nu}_{i}=\boldsymbol{\alpha}\right)=\int_{B} p_{i \boldsymbol{\alpha}}(u) G_{i}(d u), \quad B \in \mathscr{B}, \quad \boldsymbol{\alpha} \in \mathbb{N}^{n},
$$

где $\mathbb{N}$ - множество неотрицательных целых чисел. Процесс начинается с частицы, скажем, $i$-го типа, имеющей нулевой возраст в момент $t=0$. Эволюции различных частиц предполагаются независимыми друг от друга, а условное распределение эволюций всех частиц данного поколения при условии, что известны эволюции всех частищ предыдущих поколений, зависит лишь от состава этого поколения. Обозначим через $a_{i j}(u)=\sum_{\boldsymbol{\alpha} \in \mathbb{N}^{n}} \alpha_{j} p_{i \boldsymbol{\alpha}}(u)$ математическое ожидание числа частищ $j$-го типа, появляющихся в следующем поколении из одной частищы $i$-го типа, при условии, что эта частица исчезает в возрасте $u$. Тогда

$$
A_{i j}=\int_{0}^{\infty} a_{i j}(u) G_{i}(d u)
$$

- математическое ожидание числа частищ-потомков $j$-го типа у одной частищы типа $i$. Запись $\mathbf{Z}(t)=\left(Z_{i j}(t)\right)$ будет означать, что в момент $t$ существуют $Z_{i j}(t)$ частиц $j$-го типа при условии, что процесс начался с одной частицы $i$-го типа, $i, j=1, \ldots, n$.

Пусть $\mathbf{A}(t)=\left(A_{i j}(t)\right) \stackrel{\text { def }}{=} \mathbf{E Z}(t)$ - матрица соответствуюших математических ожиданий и $G_{i}(\{0\})=0 \forall i$. Тогда функции $A_{i j}(t)$ удовлетворяют следуюшему многомерному уравнению восстановления $[1$, гл. VIII, $\S 6,(7)]$ :

$$
A_{i j}(t)=\sum_{k=1}^{n} \int_{0}^{t} a_{i k}(u) A_{k j}(t-u) G_{i}(d u)+\delta_{i j}\left[1-G_{i}(t)\right], \quad i, j=1, \ldots, n,
$$

где $\delta_{i j}$ - символ Кронекера. Обозначим

$$
F_{i j}(d x) \stackrel{\text { def }}{=} a_{i j}(x) G_{i}(d x), \quad K_{i j}(x) \stackrel{\text { def }}{=} \delta_{i j}\left[1-G_{i}(x)\right], \quad X_{i j}(x) \stackrel{\text { def }}{=} A_{i j}(x) .
$$

В итоге приходим к уже исследованному многомерному уравнению восстановления (1) при $m_{1}=n$. Образуем матрицы $\mathbf{F}, \mathbf{K}$ с элементами $F_{i j}, K_{i j}$ соответственно, задаваемыми равенствами (23). Отметим, что функции $K_{i j}(x)$ суть функции 
распределения мер $K_{i j}=\delta_{i j}\left(\delta-G_{i}\right), i, j=1, \ldots, n$. Введем сокращенное обозначение для диагональных матрищ: $\operatorname{diag}\left\{a_{i}\right\} \stackrel{\operatorname{def}}{=} \operatorname{diag}\left\{a_{1}, \ldots, a_{n}\right\}$. Таким образом, $\mathbf{K}=\operatorname{diag}\left\{\delta-G_{i}\right\}$. Матрица $\widehat{\mathbf{F}}(0)=\left(\int_{0}^{\infty} a_{i j}(x) G_{i}(d x)\right)$ предполагается неприводимой.

Пусть $\widehat{\mathbf{F}}\left(r_{+}\right)<\infty$ и $\widehat{G}_{i}\left(r_{+}\right)<\infty, i=1, \ldots, n$, при $r_{+} \geqslant 0$. Как и в $\S 3$, пусть $\mathscr{Z}=\left\{s_{1}, \ldots, s_{l}\right\}-$ множество корней уравнения $\operatorname{det}(\mathbf{I}-\widehat{\mathbf{F}}(s))=0$, лежащих в полуплоскости $\left\{s \in \mathbb{C}: \operatorname{Re} s \leqslant r_{+}\right\}$, с кратностями $n_{j}, j=1, \ldots, l$. Если $\mathscr{Z} \neq \varnothing$, то, как и прежде, $q=s_{1}$ - единственный вешественный корень уравнения (3) кратности $n_{1}=1$. Пусть

$$
\int_{0}^{\infty} x^{2 n_{j}} e^{\operatorname{Re} s_{j} x} a_{i j}(x) G_{i}(d x)<\infty \quad \forall i, j
$$

и

$$
\int_{0}^{\infty} x^{n_{j}} e^{\operatorname{Re} s_{j} x}|\mathbf{K}|(d x)<\infty,
$$

где $s_{j} \in \mathscr{Z}$. Определим коэффициенты $\mathbf{B}_{j k}, k=1, \ldots, n_{j}$, из асимптотического разложения: при $s \rightarrow s_{j}$

$$
[\mathbf{I}-\widehat{\mathbf{F}}(s)]^{-1} \widehat{\mathbf{K}}(s)=[\mathbf{I}-\widehat{\mathbf{F}}(s)]^{-1} \operatorname{diag}\left\{1-\widehat{G}_{i}(s)\right\}=\sum_{k=1}^{n_{j}} \frac{(-1)^{k} \mathbf{B}_{j k}}{\left(s-s_{j}\right)^{k}}+o\left(\frac{1}{s-s_{j}}\right)
$$

ТЕОРемА 4. Пусть $\varphi(x), \quad x \in \mathbb{R},-$ полумультипликативная функиия такая, что $\varphi(x) \equiv 1$ при $x<0, \quad r_{+} \geqslant 0$ и функиия $\varphi(x) / \exp \left(r_{+} x\right), \quad x \geqslant 0$, не убывает. Предположим, что

$$
\widehat{\mathbf{F}}\left(r_{+}\right)=\left(\int_{0}^{\infty} e^{r_{+} x} a_{i j}(x) G_{i}(d x)\right)<\infty
$$

и спектральный радиус матрищь $\left(\mathbf{F}^{m *}\right)_{s}^{\wedge}\left(r_{+}\right)$меньше единиць при некотором иелом $m \geqslant 1$. Допустим, что множсество $\mathscr{Z}$ корней характеристического уравнения (3), лежсащих в полуплоскости $\left\{s \in \mathbb{C}: \operatorname{Re} s \leqslant r_{+}\right\}$, конечно. Обозначим через $N$ максимальную кратность корней уравнения (3), лежсащих на прямой $\left\{s \in \mathbb{C}: \operatorname{Re} s=r_{+}\right\}$. Если $\mathscr{Z} \neq \varnothing$, то выберем левый и правый собственные векторы $\mathbf{l}$ и $\mathbf{r}$ с положительными координатами, отвечающие собственному значению 1 матрицы $\widehat{\mathbf{F}}(q)$, так, чтобы $\mathbf{l} \mathbf{r}=1$. Пусть также

$$
\int_{0}^{\infty} x^{2 N} \varphi(x) \mathbf{F}(d x)<\infty, \quad \int_{0}^{\infty} x^{N} \varphi(x)|\mathbf{K}|(d x)<\infty .
$$

Тогда если $0 \notin \mathscr{Z}$, то

$$
\begin{aligned}
& \mathbf{A}(x)=-\sum_{j=1}^{l} \sum_{k=1}^{n_{j}} \mathbf{B}_{j k} e^{-s_{j} x} \sum_{p=0}^{k-1} \frac{x^{p}}{p ! s_{j}^{k-p}}+\boldsymbol{\rho}(x) \\
&= \begin{cases}\frac{\mathbf{r l} \operatorname{diag}\left\{\widehat{G}_{i}(q)-1\right\} e^{-q x}}{q \mathbf{l} \widehat{\mathbf{F}}^{\prime}(q) \mathbf{r}} & \text { npu } \mathscr{Z} \neq \varnothing, \\
-\sum_{j=2}^{l} \sum_{k=1}^{n_{j}} \mathbf{B}_{j k} e^{-s_{j} x} \sum_{p=0}^{k-1} \frac{x^{p}}{p ! s_{j}^{k-p}}+\boldsymbol{\rho}(x) & \text { npu } \mathscr{Z}=\varnothing, \\
\boldsymbol{\rho}(x) & \end{cases}
\end{aligned}
$$


əдe

$$
|\boldsymbol{\rho}(x)| \leqslant \operatorname{Var}\{\boldsymbol{\rho}(y) ; y \in(x, \infty)\}=o(1 / \varphi(x)) \quad \text { nрu } x \rightarrow \infty .
$$

А если $q=0 \in \mathscr{Z}$, mo

$$
\mathbf{A}(x)=\frac{\mathbf{r l} \operatorname{diag}\left\{\int_{0}^{\infty} x G_{i}(d x)\right\}}{\mathbf{l} \widehat{\mathbf{F}}^{\prime}(0) \mathbf{r}}-\sum_{j=2}^{l} \sum_{k=1}^{n_{j}} \mathbf{B}_{j k} e^{-s_{j} x} \sum_{p=0}^{k-1} \frac{x^{p}}{p ! s_{j}^{k-p}}+\boldsymbol{\rho}(x),
$$

где $\boldsymbol{\rho}(x)$ удовлетворяет соотношению (25).

ДокаЗАТЕЛЬСТво. Пусть $0 \notin \mathscr{Z}$. Применим следствие 1 . Положим $\boldsymbol{\rho}(x)=$ $-\boldsymbol{\Delta}_{\mathbf{K}}((x, \infty))$. В нашем случае $\widehat{\mathbf{K}}(0)=\operatorname{diag}\left\{1-\widehat{G}_{i}(0)\right\}=\mathbf{0}$. Соотношения (24) и $(25)$ установлены. Пусть теперь $q=0 \in \mathscr{Z}$. Тогда достаточно применить следствие 2. Положим $\boldsymbol{\rho}(x)=-\boldsymbol{\Delta}_{\mathbf{K}}((x, \infty))$. В данном случае $\widehat{\mathbf{K}}(0)=\mathbf{0}$ и $\widehat{\mathbf{K}}^{\prime}(0)=$ $\operatorname{diag}\left\{-\widehat{G}_{i}^{\prime}(0)\right\}=-\operatorname{diag}\left\{\int_{0}^{\infty} x G_{i}(d x)\right\}$. Теорема доказана.

ЗАмЕчАниЕ 2 . Если функции $a_{i j}(x)$ ограничены, то моментные условия теоремы 3 можно заменить на $\int_{0}^{\infty} x^{2 N} \varphi(x) G_{i}(d x)<\infty \forall i$.

\section{§5. Сравнение результатов}

В заключение подчеркнем универсальность теорем и следствий настоящей работы, позволяюших получить ряд известных результатов в качестве весьма частных случаев. В терминологии [1, гл. VIII, § 9] уравнение (2) называется критическим, если перронов корень матрицы $\widehat{\mathbf{F}}(0)$ равен единице (иными словами, если $\left.q=s_{1}=0\right)$. В теоремах 1,2 и 4 из [1, гл. VIII, § 9] рассматривается критический случай и предполагается, что все элементы матрицы $\mathbf{F}-$ абсолютно непрерывные меры, что автоматически влечет $\mathbf{F}_{s}=\mathbf{0}$, и, следовательно, спектральный радиус матрицы $\mathbf{F}_{s}^{\wedge}(0)$ равен нулю. Пусть $L$ - сужение меры Лебега на $[0, \infty)$. В наших обозначениях теорема 1 из [1, гл. VIII, § 9] гласит, что если $\int_{0}^{\infty} x^{2} \mathbf{F}(d x)<\infty$, то $\int_{0}^{\infty}\left|\mathbf{H}-\delta \mathbf{I}-\mathbf{B}_{11} L\right|(d x)<\infty$, а если дополнительно предположить $\int_{0}^{\infty} x^{3} \mathbf{F}(d x)<\infty$, то $\int_{0}^{\infty} x\left|\mathbf{H}-\delta \mathbf{I}-\mathbf{B}_{11} L\right|(d x)<\infty$. Процитированньй результат получается из теоремы 3 при $\mathbf{K}=\mathbf{F}, N=1, \varphi(x) \equiv 1$ в первом случае и $\varphi(x)=1+x, x \geqslant 0$, во втором. Если $\int_{0}^{\infty} x^{3} \mathbf{F}(d x)<\infty$, то теорема 3 из [1, гл. VIII, $\S 9]$ гласит, что $\mathbf{X}(x)=\mathbf{B}_{11} x+\mathbf{B}_{0}+o(1)$ при $x \rightarrow \infty$, где $\mathbf{B}_{0}$ - вполне определенная матрица. Следствие 2 в этих условиях усиливает данное утверждение: $o(1)$ можно заменить на $o(1 / x)$; для этого достаточно в следствии 2 положить $\varphi(x)=1+x$ и $N=1$. А результат с $o(1)$ получается уже при $\int_{0}^{\infty} x^{2} \mathbf{F}(d x)<\infty$, в этом случае в следствии 2 возьмем $\varphi(x) \equiv 1$.

В теореме 4 из [1, гл. VIII, $\S 9]$ говорится, что если $\int_{0}^{\infty} x^{3} \mathbf{F}(d x)<\infty$, a $\mathbf{K}(x) \downarrow 0$ при $x \rightarrow \infty$ и $\int_{0}^{\infty} x \mathbf{K}(x) d x<\infty$, то (в наших обозначениях)

$$
\mathbf{X}(x)=\frac{\mathbf{r l} \widehat{\mathbf{K}}^{\prime}(0)}{\mathbf{l} \widehat{\mathbf{F}}^{\prime}(0) \mathbf{r}}+o\left(\frac{1}{x}\right), \quad x \rightarrow \infty .
$$

Этот результат получается из следствия 2 при $\varphi(x)=1+x$ и $N=1$.

При исследовании асимптотики моментов зависяших от возраста ветвяшихся процессов в [1] предполагается, что все меры $F_{i j}(d x)=a_{i j}(x) G(d x)$ абсолютно 
непрерывны, что автоматически влечет $\mathbf{F}_{s}=\mathbf{0}$. Перейдем к сравнению результатов, касающихся асимптотики среднего числа частиц ветвящихся процессов. Теорема 1 из [1, гл. VIII, § 10] гласит, что если процесс критический (т.е. $q=s_{1}=0$ ), если $\int_{0}^{\infty} x^{r} G_{i}(d x)<\infty \forall i$ и $\int_{0}^{\infty} x^{r+1} \mathbf{F}(d x)<\infty$, то $\mathbf{A}(x)=\mathbf{A}_{0}+o\left(x^{1-r}\right)$ при $x \rightarrow \infty$, где $r=1,2$. Этот результат вытекает из следствия 2 при $\mathbf{K}=\operatorname{diag}\left\{\delta-G_{i}\right\}$ и $\varphi(x)=(1+x)^{r-1}$ (в данном случае $\left.N=1\right)$.

ЗАмЕчАниЕ 3 . Пусть $\varphi(k), k \in \mathbb{Z},-$ полумультипликативная функция, определенная на множестве целых чисел $\mathbb{Z}$. Если в доказательствах теорем и следствий заменить преобразования Лапласа на производящие функции и вместо алгебр $S(\varphi)$ использовать банаховы алгебры комплексных последовательностей $\left\{\nu_{k}\right\}$ таких, что $\sum_{k=-\infty}^{\infty} \varphi(k)\left|\nu_{k}\right|<\infty$, то получим аналоги соответствующих утверждений для мер и распределений, сосредоточенных на множестве $\mathbb{Z}$. При этом в формулировки теорем необходимо внести соответствующие изменения: надо заменить меры $L, \mathscr{E}_{j}$ на их дискретные аналоги. Кроме того, при рассмотрении дискретного случая нет надобности в условии на спектральный радиус матрищы $\left(\mathbf{F}^{m *}\right)_{s}^{\wedge}\left(r_{+}\right)$.

\section{Список литературы}

1. Севастьянов Б. А. Ветвящиеся процессы. М.: Наука, 1971.

2. Athreya K.B., Ney P. A Markov process approach to systems of renewal equations with application to branching processes // Branching processes. Advances in Probability and Related Topics. V. 5. N. Y.: Marcel Dekker, 1978. P. 293-317.

3. Севастьянов Б. А., Чистяков В.П. Уравнения многомерного восстановления и моменты ветвящихся процессов // Теория вероятностей и ее применения. 1971. Т. 16. № 2 . C. 201-217.

4. Crump K.S. On systems of renewal equations // J. Math. Anal. Appl. 1970. V. 30. P. $425-434$.

5. Crump K.S. On systems of renewal equations: the reducible case // J. Math. Anal. Appl. 1970. V. 31. P. 517-528.

6. Ланкастер П. Теория матриц. М.: Наука, 1982.

7. Хилле Э., Филлипс P. Функциональный анализ и полугруппы. М.: ИЛ, 1962.

8. Феллер В. Введение в теорию вероятностей и ее приложения. Т. 2. М.: Наука, 1967.

9. Sgibnev M. S. Submultiplicative moments of the supremum of a random walk with negative drift // Statist. Probab. Lett. 1997. V. 32. P. 377-383.

10. Sgibnev $M$. S. An asymptotic expansion for the distribution of the supremum of a random walk // Studia Math. 2000. V. 140. P. 41-55.

11. Jelenković P. R., Lazar A. A. Subexponential asymptotics of a Markov-modulated random walk with queueing applications // J. Appl. Probab. 1998. V. 35. P. 325-347.

12. Хорн Р., Джсонсон Ч. Матричный анализ. М.: Мир, 1989.

13. Рогозин Б. А., Сзибнев M. C. Банаховы алгебры мер на прямой // Сиб. матем. журн. 1980. T. 21. № 2. C. 160-169.

630090, г. Новосибирск,

Институт математики им. С. Л. Соболева СО РАН

Поступило в редакцию

23.II. 2001 